\title{
Auditory apparent motion under binaural and monaural listening conditions
}

\author{
THOMAS Z. STRYBEL \\ California State University, Long Beach, California \\ and \\ CAROL L. MANLIGAS and DAVID R. PERROTT \\ California State University, Los Angeles, California
}

\begin{abstract}
This investigation examined the ability of listeners to perceive apparent motion under binaural and monaural listening conditions. Fifty-millisecond broadband noise sources were presented through two speakers separated in space by either $10^{\circ}, 40^{\circ}$, or $160^{\circ}$, centered about the subject's midline. On each trial, the sources were temporally separated by 1 of 12 interstimulus onset intervals (ISOIs). Six listeners were asked to place their experience of these sounds into one of five categories (single sound, simultaneous sounds, continuous motion, broken motion, or successive sounds), and to indicate either the proper temporal sequence of presentation or the direction of motion, depending on whether or not motion was perceived. Each listener was tested at all spatial separations under binaural and monaural listening conditions. Motion was perceived in the binaural listening condition at all spatial separations tested for ISOIs between 20 and $130 \mathrm{msec}$. In the monaural listening condition, motion was reliably heard by all subjects at $10^{\circ}$ and $40^{\circ}$ for the same range of ISOIs. At $160^{\circ}$, only 3 of the 6 subjects consistently reported motion. However, when motion was perceived in the monaural condition, the direction of motion could not be determined.
\end{abstract}

An observer of a moving object can obtain information about the object's motion through several sense modalities, yet those who study motion perception treat it almost exclusively as a visual phenomenon. For example, until very recently, investigators of auditory space perception have ignored the question of moving sounds.

Interest in visual motion perception has persisted for over a century. The illusions of motion have been of particular interest, because they pose a problem for theories of visual motion perception. Prior to their discovery, the perception of motion was easily explained: If an object in space stimulates a particular location on the retina, one should be able to perceive continuous changes in position on the retina as motion. The illusions of motion-in particular, apparent motion-can produce the sensation of movement from the stimulation of only two separate points on the retina, and thus the "problem" for motion perception (Boring, 1942).

Since Wertheimer's (1912) seminal report on visual apparent motion, literally hundreds of articles have been published on the topic. Kolers (1972) noted that there are more reports of illusory motion than on real motion per-

This research was supported in part by grants from the National Science Foundation (BNS-8512317) and the National Institute of Health (3S06 RR0801-1452). Carol L. Manligas is now at the Department of Psychology, University of Georgia, Athens, GA 30602. David L. Perrott is with the Psychoacoustics Laboratory, Department of Psychology, California State University, Los Angeles, CA 90032. Address reprint requests to Thomas Z. Strybel, Department of Psychology, California State University, Long Beach, CA 90840. ception. He attributed this to the assumption that real and illusory motion are mediated by similar processes. However, the fact that illusory movement is simply easier to produce must have also influenced the choice of stimuli (for an overview of the visual apparent motion literature, see Anstis, 1978).

\section{Auditory Apparent Motion}

Five years after Wertheimer's publication, Burtt (1917) demonstrated that apparent motion can be produced in the auditory modality. Since that time, very little attention has been paid to the auditory phenomenon. The majority of the work on auditory apparent motion has investigated the similarities between the auditory and visual illusions.

Burtt (1917) demonstrated that for interstimulus intervals (ISIs) near $0 \mathrm{msec}$, two simultaneous images were reported. As the ISI increased, first continuous motion, then broken motion, and finally succession were reported by the majority of his subjects. Hisata (1934) replicated these findings, but Matheissen (1931) failed to elicit reports of apparent motion from the majority of her subjects.

Briggs and Perrott (1972) showed that the relationship between the duration of the stimulus and the ISI was similar to that found in the visual modality by Korte (1915). As the duration increases, the ISI that produces optimal movement reports decreases. Perrott (1974) showed that neither changes in the rise time of the stimuli nor changes in the correlation between the signal microstructure affected the ISI that produced apparent motion. 
The major difference between visual and auditory apparent motion found in all of these reports is in the ISI values that produce movement. In audition, apparent motion is heard at ISIs that are lower than the ISIs required for visual apparent motion, and can be heard with considerable temporal overlap. For example, Briggs and Perrott (1972) determined that motion is perceived for interstimulus onset intervals (ISOIs) between 10 and $90 \mathrm{msec}$ for a pulse duration of $50 \mathrm{msec}(90 \mathrm{msec}$ was the largest ISOI tested). This, of course, corresponds to ISI values from -40 to $40 \mathrm{msec}$. These ISOIs are considerably lower than the range of ISIs reported by Neuhaus (1930) in vision (60 to $270 \mathrm{msec}$ for $40-\mathrm{msec}$ duration).

\section{Simulated Motion}

Although interest in the illusion of auditory apparent motion has been minimal, the problem of auditory motion perception has been examined with simulated motion. Simulated motion is an attractive alternative to real motion because of the difficulty in physically moving a sound source without adding any extraneous noise. Simulated motion has been obtained by reproducing the dynamic interaural differences that result from a moving sound source. Two methods have been reported. One method presents dichotic click trains, at first with no interaural differences. Successive clicks are presented with increasing interaural differences, and the sound appears to move from the center of the head to the ear receiving the earlier pulse (Altman, 1968; Altman \& Viskov, 1977; Viskov, 1975). A second technique allows free field testing by initially simulating the position of a sound source located between two fixed speakers according to the law of sines (Bauer, 1961). "Motion" is produced by dynamically changing the relative levels of the signals arriving to each speaker. This technique involves fading off one signal while fading on the second (Grantham, 1986).

Viskov (1975) performed the first psychophysical investigation of simulated movement. Viskov investigated the conditions under which the dichotic click technique produced the sensation of movement. For example, the minimum click frequency that produced movement varied between 4.8 and $9.2 \mathrm{~Hz}$, with the average threshold corresponding to $7.6 \mathrm{~Hz}$. Additional experiments showed that the intensity of the click train had no effect on the threshold; the minimum stimulus duration that produced movement of the sound image ranged from 100 to $150 \mathrm{msec}$; and the minimum number of clicks required was two. Only 1 of 3 subjects showed a decrease in threshold frequency as the duration increased from 150 to 4,800 msec. Viskov concluded that the dichotic click technique would be sufficient for the investigation of auditory motion perception.

Altman and Viskov (1977) subsequently used the dichotic click technique to measure velocity discrimination. Velocity difference limens were found to increase from $10.8^{\circ}$ to $19.3^{\circ}$ as the velocity of the simulated moving image increased from $14^{\circ}$ to $140^{\circ} / \mathrm{sec}$. Grantham (1986) used the simulated movement of a $500-\mathrm{Hz}$ tone to measure both displacement thresholds and velocity discrimination in the free field. The displacement threshold, called the minimum audible movement angle, was shown to increase with the velocity of the source. The velocity threshold also increased with source velocity, and the results agreed with those obtained by Altman and Viskov (1977).

Although simulated motion is an attractive procedure for the investigation of auditory motion perception, it is probably a variation on the illusion of apparent motion. Viskov's (1975) results illustrate this observation most clearly. Viskov observed that a minimum of two dichotic clicks are required for the perception of motion, which corresponds to the classic method of producing apparent motion. Furthermore, the minimum click frequency $(7.6 \mathrm{~Hz})$ required for motion to be reported corresponds to an ISOI of $132 \mathrm{msec}$, well within the range required for apparent motion. The technique employed by Grantham (1986) might also be described in this manner. In his experiments, changes in the intensity of each speaker occurred at an update rate of $20-500 \mathrm{~Hz}$. Therefore, sound "images" at different locations in space were presented at ISOIs between 5 and $20 \mathrm{msec}$. Although the lower value is below the range reported for apparent motion, it is possible that his listeners were unable to distinguish between each image presented. Simulated motion may involve the proper timing of sound images, as opposed to the timing of actual sound sources used in traditional auditory apparent motion research.

Thus, simulated motion may be another instance of apparent motion. However, these simulations have replicated only the interaural differences in time or intensity produced by a moving sound source. Any influence of monaural spectral changes has not been examined. The present study was conducted for this reason. An apparent motion paradigm was used to determine if the illusion of auditory apparent motion could be heard under both binaural and monaural listening conditions.

\section{METHOD}

\section{Subjects}

Six subjects participated in this experiment, 3 of them the authors. All subjects had participated in previous apparent motion experiments and had normal hearing.

\section{Apparatus}

Testing was conducted in an audiometric chamber with the walls modified to produce a semi-anechoic environment. This modification consisted of attaching Sonex acoustic foam wedges (NSX-4) to the walls, ceiling, and floor of the room. A chair was positioned in the center of the room. Mounted to the back of the chair was a vertical pole. Attached to the top of this pole were two beams, mounted so that they could rotate in a horizontal plane above the subject's head. This arrangement resembled a chair with a large propeller attached to the top, except that each "blade" of the propeller could be individually rotated by hand. Three-inch speakers were hung from these beams. These speakers could be positioned at any azimuth angle around the subject, and at any distance from the subject up to $1 \mathrm{~m}$. A modified helmet was hung from the center axis of rotation. The helmet served several purposes. It ensured 
that the head of the subject was in the center of the axis of rotation, it enabled the speakers to be adjusted vertically until they were at ear level, and it limited movements of the head during testing. The helmet did not extend below the pinnae of the subject.

Stimulus presentation and response collection were controlled by electronic programmable modules in conjunction with a microprocessor. Broadband noise $(1000-8000 \mathrm{~Hz})$ was led from two noise generators, through bandpass filters into separate electronic switches, and finally to the speakers. Responses were collected by a response box connected to the game port of the computer. The box contained five three-position switches, which corresponded to the response categories available to the subject.

Monaural listening conditions were produced by fitting the subject's left ear with a Hear Saver earplug. These plugs reportedly provide attenuation of $40 \mathrm{~dB}$ for frequencies above $1000 \mathrm{~Hz}$. An earmuff was also placed over the left ear, and the noise sources were filtered to allow only frequencies above $1000 \mathrm{~Hz}$. A test of the monaural conditions was made on one subject (D.R.P.). Both ears were fitted with ear stopples and earmuffs, and his absolute threshold was determined. This value, when compared with the threshold obtained with both ears open, was in fact $40 \mathrm{~dB}$ higher in the predicted frequency range.

\section{Procedure}

The stimulus consisted of two 50-msec noise bursts (40 dBA weighted with rise time of $.1 \mathrm{msec}$ ) which were led to each speaker and separated in time by one of 12 ISOIs $(0,10,20,30,45,75$, $90,105,130,200,300$, or $500 \mathrm{msec})$. Each ISOI value was presented 30 times at each speaker position, half of them originating in the speaker to the right of the subject, and half to his/her left. A trial block therefore consisted of 360 trials presented to the subject at a particular speaker position in random order.

Three different values of spatial separation were tested $\left(10^{\circ}, 40^{\circ}\right.$, and $160^{\circ}$, centered about the midline of the listener) under both monaural and binaural listening conditions. Thus, six sessions were run. All trials were run at one condition and separation before changing to the next in random sequence.

The subject was instructed to listen to the sound presentation and classify his/her perception of the stimulus into one of five categories. ${ }^{1}$ The categories were single sound (a single sound image was heard somewhere, with no perception of movement), simultaneous sounds (two sounds were heard at the same time at two different locations, with no movement between them), continuous motion (a sound was heard at one side and moved continuously toward the other side), broken movement (the sound was heard to move from one side to the other, but the movement was interrupted or discontinuous), and successive sounds (two sounds were heard, one at one location, then another at a different location, with no movement between them).

In addition to classifying the perception, the subject indicated whether the sound originated on his/her left or right. When no motion was perceived, this was a judgment of the temporal order of presentation (e.g., did the first sound originate on the right or the left?). Of course, when the "single" or "simultaneous" responses were made, the subject was forced to guess at the correct order. When motion was heard, the subject could judge the position of the lead source by detecting the direction of motion (e.g., whether the sound traveled from left to right, or vice versa). The category was chosen by selecting the appropriately labeled switch on the response box, which was held in the hand of the subject. The position of the lead source was indicated by throwing the switch to either the left or the right.

Prior to the initial trial block, the subject read the instructions and was allowed to ask the experimenter any questions regarding the categories of response. At the beginning of each subsequent session, the subject was allowed to review the response categories. When a light, located on the response box, was illuminated, the subject could initiate the session by throwing any switch. The light was turned off, and the first stimulus pair was presented $500 \mathrm{msec}$ later. The light on the box was again illuminated $500 \mathrm{msec}$ after the stimulus presentation, indicating to the subject to make his/her response. The light was turned off after the response was made, and $500 \mathrm{msec}$ later the next stimulus pair was presented. Thus the trial blocks were self-paced in that the subject could delay the onset of a subsequent trial by delaying the response entry.

\section{RESULTS}

Figures 1-3 present the distribution of mean responses at all separations tested, both for monaural and binaural listening conditions. For the binaural listening condition at $10^{\circ}$ separation (Figure $1 \mathrm{~A}$ ), the dominant response for ISOIs of less than $20 \mathrm{msec}$ was "simultaneous." For ISOIs between 20 and $45 \mathrm{msec}$, "continuous motion" was most often reported, followed by "broken motion" (75-130 msec), and finally by "succession." In the monaural condition (Figure 1B), the dominant response at small ISOIs was "single." For larger ISOIs the distribution was nearly identical to the binaural condition. Although the peaks of the curves are smaller in the monaural condition, the range of ISOIs that produce motion on the average are identical to the binaural condition.

These similarities are repeated at $40^{\circ}$ separation, as shown in Figure 2. The motion curves differ only in the
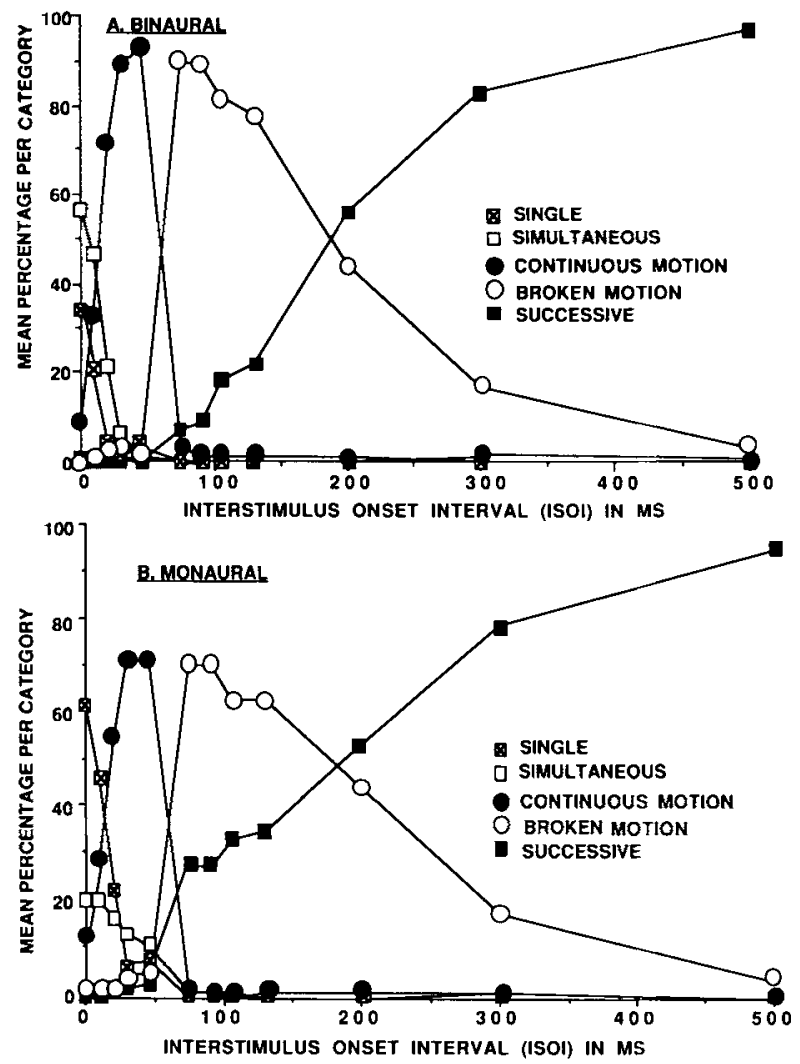

Figure 1. Mean percentage of responses in each category over the range of interstimulus onset intervals tested for a spatial separation of $10^{\circ}$ during (A) binaural listening and (B) monaural listening. 
magnitude of the peaks. In the binaural condition at $160^{\circ}$ separation (Figure 3A), fewer "continuous motion" responses were made, although the range of ISOIs that produced this response was similar to lesser separation values. For monaural listening conditions, the "continuous motion" and "broken motion" responses were greatly reduced. In fact, the mean percentage of "continuous motion" reports barely exceeded "single" reports at only one ISOI ( $45 \mathrm{msec})$. Although the mean performance shown here suggests that motion is not reliably detected, considerable intersubject variability was found, as discussed below.

These results suggest little or no effect of separation on apparent motion in the binaural condition and an overall decrease in motion reports with increases in separation in the monaural condition. This is illustrated in Figure 4, which provides the percentage of motion reports ("continuous" + "broken") as a function of ISOI, for all conditions tested. Although the shapes of the curves are nearly identical, fewer motion reports on the average were given for monaural listening conditions.

Considerable intersubject variability was found in the monaural listening condition. For example, one subject (O.C.) reported almost no motion of any kind in the monaural condition. This subject also had difficulty perceiving motion binaurally on practice trials. Of the re-
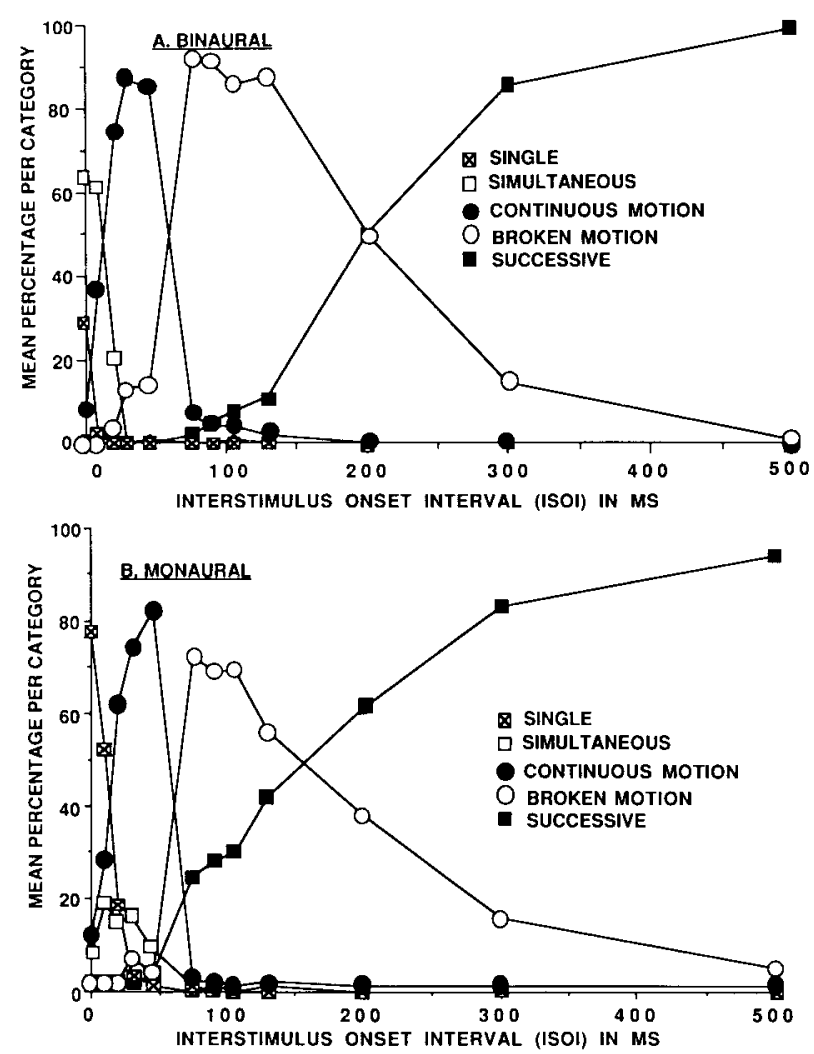

Figure 2. Mean percentage of responses in each category over the range of interstimulus onset intervals tested for a spatial separation of $40^{\circ}$ during (A) binaural listening and (B) monaural listening.
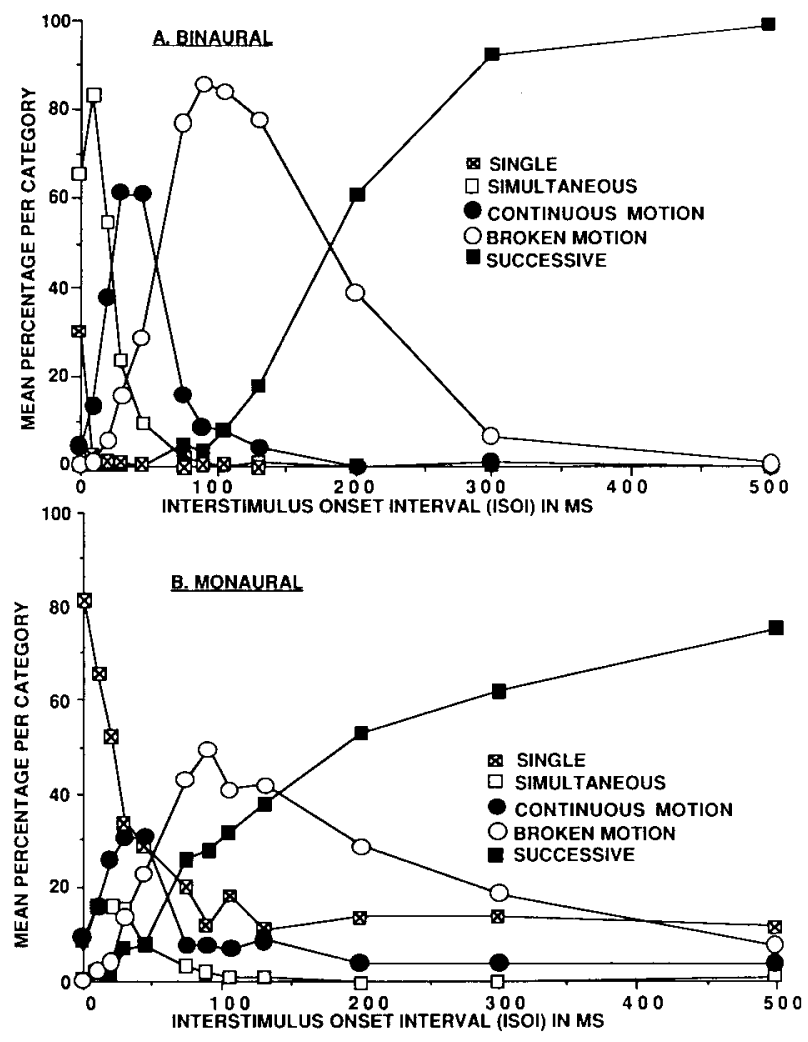

Figure 3. Mean percentage of responses in each category over the range of interstimulus onset intervals tested for a spatial separation of $160^{\circ}$ during $(A)$ binaural listening and $(B)$ monaural listening.

maining 5 subjects, only 2 (T.Z.S. and D.R.P.) reported less motion at larger values of spatial separation in the monaural listening condition. The remaining three had motion curves that were identical to their binaural functions at all values of separation.

The mean percentage of correct order judgments are shown in Figure 5. For binaural listening, the percentage correct was not affected by the separation, reaching greater than chance levels (75\%) between 10 and $20 \mathrm{msec}$. In the monaural listening condition, separation again appeared to affect performance. At the largest value of separation, performance exceeded chance, on the average, at an ISOI of $130 \mathrm{msec}$. At $40^{\circ}$ the mean percentage did not exceed chance until the ISOI reached $300 \mathrm{msec}$. At a separation of $10^{\circ}$, performance was well below chance levels at all separations tested. The results in the monaural condition were again highly variable, however. One subject was able to exceed chance levels at $10^{\circ}$. Four subjects exceeded chance performance at $40^{\circ}$, and 5 subjects exceeded chance levels at $160^{\circ}$.

\section{DISCUSSION}

This experiment demonstrated that listeners can perceive the illusion of apparent motion in the absence of dynamic interaural differences. However, under monaural 


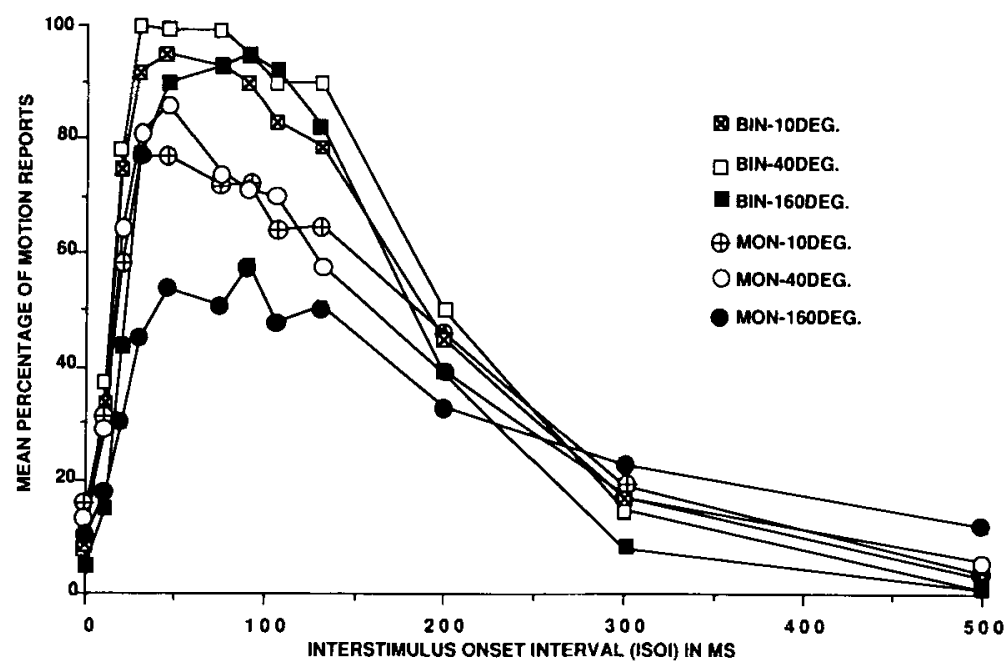

Figure 4. Mean percentage of motion reports ("continuous motion" + “broken motion") under binaural and monaural listening conditions, for all separations and interstimulus onset intervals tested.

listening conditions, the direction of motion was not accurately detected. Only at a separation of $160^{\circ}$ did average performance of correct temporal order exceed chance levels across a wide range of ISOIs; however, at this separation, motion was rarely reported.

It appears that neither the relationship between separation and ISOI asserted by Korte (1915) nor that asserted by Neuhaus (1930) for the visual illusion can be applied to the auditory binaural function. Korte asserted that the ISI that produces visual apparent motion must increase as the separation increases. Neuhaus indicated that the range of ISIs decreases as the separation increases. In the binaural listening condition, spatial separation had no ef- fect on the ISOI range that elicits motion reports. However, the monaural functions do suggest some limit on the maximum value of separation for which apparent motion can be heard, although this value may vary considerably between subjects.

The considerable intersubject variability is not surprising. Kolers (1972) described several subject variables that affect the perception of apparent motion in the visual modality. In addition, Grantham (1986) reported considerable intersubject differences for simulated motion thresholds. The variability noted in this investigation may be related to reported differences in the perceived length of the path of motion in the monaural condition. Gener-

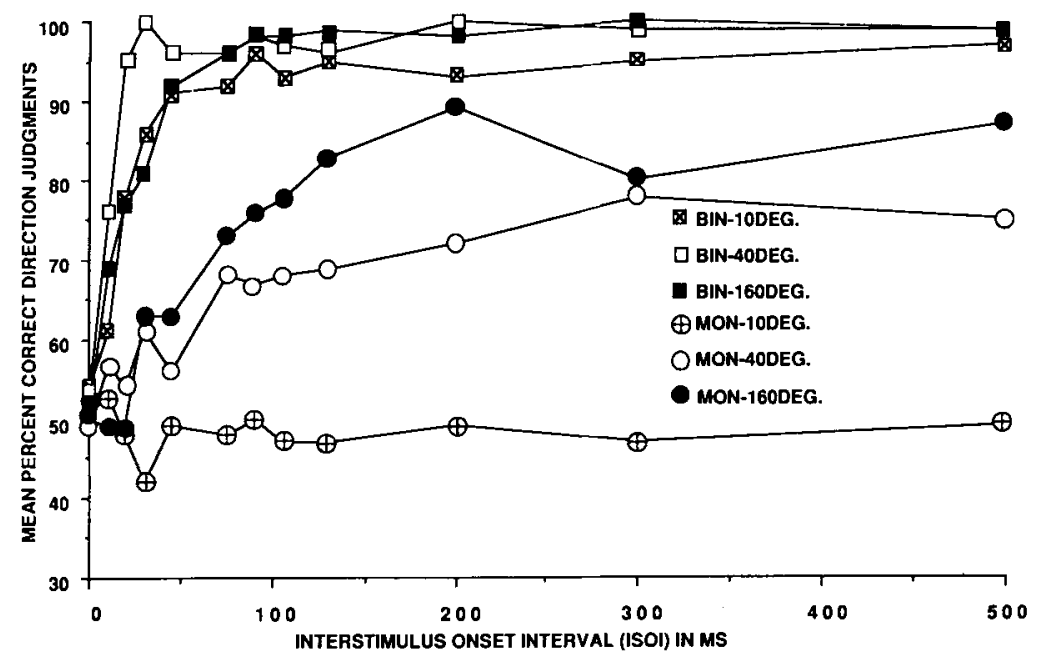

Figure 5. Mean percentage of correct direction judgments under binaural and monaural listening conditions for all separations and interstimulus onset intervals tested. 


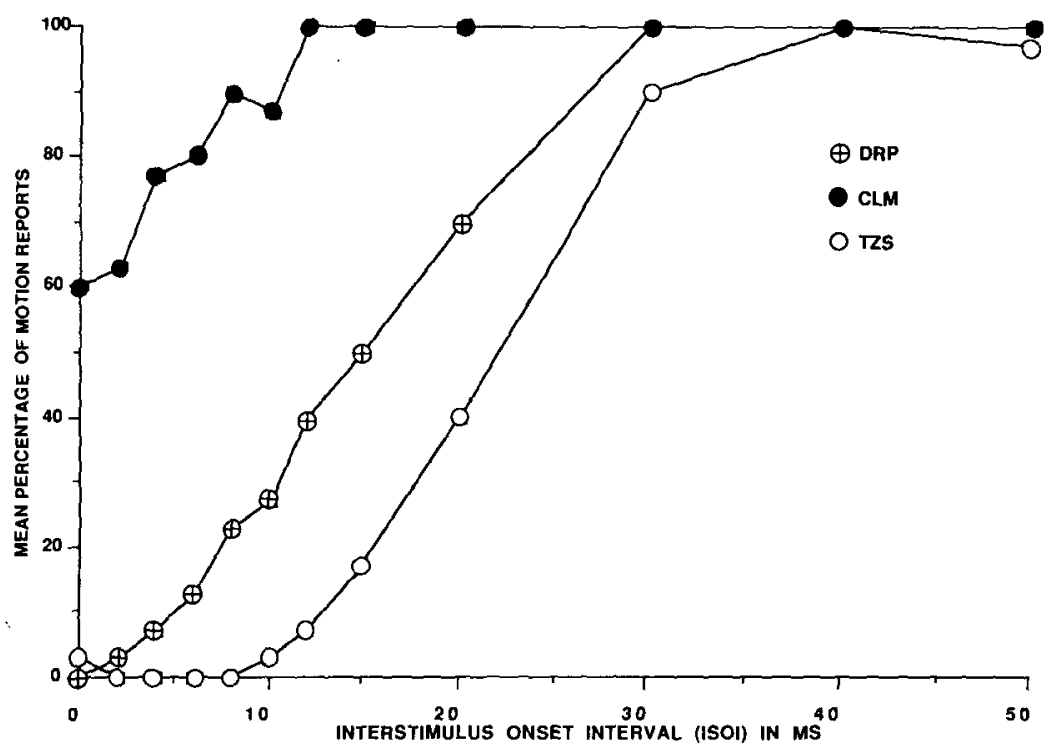

Figure 6. Mean percentage of motion reports ("continuous motion" + "broken motion $\left.{ }^{m}\right)$ under monaural listening conditions with a spatial separation of $90^{\circ}$ centered at $90^{\circ}$ azimuth ( 3 subjects).

Ally, the apparent distance traveled was shorter in the monaural condition, and the motion was almost always heard on the side adjacent to the open ear of the subject. No systematic effects of stimulus order of presentation (left to right or vice versa) were noted on either percentage of motion reports or correct direction performance, however.

One possible explanation for the difficulty in detecting the direction of motion in the monaural condition is that the speakers were not located in regions that provide optimal monaural localization. To test this interpretation, we ran additional sessions on 3 subjects in the monaural listening condition. The speakers were separated by $90^{\circ}$, and the midpoint was moved to $90^{\circ}$ azimuth. In addition, a smaller range $(0-50 \mathrm{msec})$ of ISOI values was tested to eliminate the possibility of subjects' learning a response to particular stimulus combinations. The percentage of motion reported is shown in Figure 6. Two of 3 subjects showed nearly identical functions to their binaural performance. The 3rd subject (C.L.M.) responded more frequently with the motion category at all ISOIs tested, although her function also increased with ISOI. None of these subjects was able to exceed chance levels on the direction of motion task, however. Improving the accuracy of monaural position information did not improve performance on the direction of motion task. However, it is possible to further improve the accuracy of monaural position information through the addition of higher frequency components to the stimulus. The monaural hypothesis cannot be completely rejected at the present time.

In summary, apparent motion was demonstrated under monaural listening conditions, but the direction of this motion could not be appreciated. Only in the binaural listen- ing condition could the direction of motion be determined. It appears that binaural information is not necessary for the perception of motion in the auditory modality. Probably the most extreme interpretation of these results would be that two motion detection systems exist in the auditory modality: a system that detects motion per se, and a system that detects motion by noticing changes in the position of a sound image, through the use of changing interaural stimulation. A similar distinction has been made in the visual modality (Bonnett, 1975, 1977). Of course, a great deal of additional evidence is required to substantiate such a claim.

Regardless of the interpretation, one implication of these findings is that simulations of motion for the purpose of investigating auditory motion perception should not be limited to replications of dynamic interaural time and intensity differences. Clearly, some monaural component must also be made available to the listener.

\section{REFERENCES}

Altman, J. A. (1968). Are there neurons detecting direction of sound source motion? Experimental Neurology, 22, 13-25.

Altman, J. A., \& VISKov, O. V. (1977). Discrimination of perceived movement velocity for fused auditory image in dichotic stimulation. Journal of the Acoustical Society of America, 61, 816-819.

ANSTrS, S. M. (1978). Apparent movement. In R. Held, W. Leibowitz, \& H. Teuber (Eds.), Handbook of sensory physiology: Vol. 8. Perception. Berlin: Springer-Verlag.

BAuER, B. B. (1961). Phasor analysis of some stereophonic phenomenon. Journal of the Acoustical Society of America, 33, 1536-1539.

BonNETT, C. (1975). A tentative model for visual motion detection. Psychologia, 18, 35-50.

BONNETT, C. (1977). Visual motion detection models: Features and frequency filters. Perception, 6, 491-500.

BorING, E. G. (1942). Sensation and perception in the history of experimental psychology. New York: Appleton-Century-Crofts. 
Briggs, R., \&errott, D. R. (1972). Auditory apparent movement under dichotic listening conditions. Journal of Experimental Psychology, 92, 83-91.

BurT, H. E. (1917). Auditory illusions of movement-A preliminary study. Journal of Experimental Psychology, 2, 63-75.

Grantham, D. W. (1986). Detection and discrimination of simulated motion of auditory targets in the horizontal plane. Journal of the Acoustical Society of America, 79, 1939-1949.

HisATA, T. (1934). Experimentelle Untersuchungen über die Scheinbewegungen in akustischen Gebeit. Japanese Journal of Psychology, 9, 25-26.

Kolers, P. A. (1972). Aspects of motion perception. Oxford: Pergamon Press.

KoRTE, A. (1915). Kinematoskopische Untersuchungen. Zeitschriff für Psychologie, 72, 193-206.

Matheissen, A. (1931). Apparent movement in auditory perception. Psychological Monographs, 41, 74-131.

Neuhaus, W. (1930). Experimentelle untersuchung der Scheinbewegung. Pflügers Archiv für die Gesamte Psychologie des Menschen und der Tiere, 75, 415-458.

Perrott, D. R. (1974). Auditory apparent motion. Journal of Auditory Research, 14, 163-169.
VISKov, O. V. (1975). The perception of the fused auditory image movement. Human Physiology, 1, 371-376.

Wertheimer, M. (1912). Experimentelle Studien über das Sehen von Bewegung. In T. Shipley (Ed. and Trans.), Classics in psychology. New York: Philosophical Library.

\section{NOTE}

1. These categories were developed by Briggs and Perrott (1972) in an investigation of dichotic apparent motion. The categories and their descriptions were derived from unsolicited reports of 13 subjects observed in preliminary tests of this phenomenon. The only modification made in the present investigation was to substitute the category "single" for the category "center" employed by Briggs and Perrott; this was to allow the subject to respond to an impression of a single sound image anywhere in space.

(Manuscript received November 9, 1987; revision accepted for publication September 15, 1988.) 